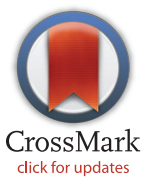

RESEARCH ARTICLE

\title{
Association of Lp-PLA 2 Mass and Aysmptomatic Intracranial and Extracranial Arterial Stenosis in Hypertension Patients
}

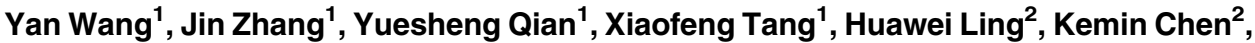 \\ Pingjin Gao', Dingliang Zhu ${ }^{1}$ * \\ 1 Research Center for Hypertension Management and Prevention in Community, Shanghai Key Laboratory \\ of Hypertension, Shanghai Institute of Hypertension, State Key Laboratory of Medical Genomics, Ruijin \\ Hospital, Shanghai Jiaotong University School of Medicine, Shanghai, China, 2 Department of Radiology, \\ Ruijin Hospital, Shanghai Jiaotong University School of Medicine, Shanghai, China \\ *zhudingliang@sibs.ac.cn
}

\section{Abstract}

\section{f open Access}

Citation: Wang Y, Zhang J, Qian Y, Tang X, Ling H, Chen K, et al. (2015) Association of Lp-PLA ${ }_{2}$ Mass and Aysmptomatic Intracranial and Extracranial Arterial Stenosis in Hypertension Patients. PLoS ONE 10(6): e0130473. doi:10.1371/journal. pone. 0130473

Editor: Xiaoying Wang, Massachusetts General Hospital, UNITED STATES

Received: March 16, 2015

Accepted: May 20, 2015

Published: June 22, 2015

Copyright: $\odot 2015$ Wang et al. This is an open access article distributed under the terms of the Creative Commons Attribution License, which permits unrestricted use, distribution, and reproduction in any medium, provided the original author and source are credited.

Data Availability Statement: All relevant data are within the paper.

Funding: This project was supported by grants from the National Key Program for Basic Research (2009CB521905), National Natural Science Foundation of China (81000037 and 81370205), National High Technology Research and Development Program (2006AA02Z179), and Shanghai Hospital Development Center Foundation (SHDC12012304). The funders had no role in study design, data collection and analysis, decision to publish, or preparation of the manuscript.

\section{Background and Purpose}

Intracranial arterial stenosis (ICAS) is a common cause of ischemic stroke in Asians, whereas whites tend to have more extracranial lesions. Lipoprotein-associated phospholipase $A_{2}\left(L p-P L A_{2}\right)$ has been associated with ischemic stroke by a large amount of work. However, there are few studies focusing on the relationship of Lp-PLA $A_{2}$ and asymptomatic ICAS or extracranial arterial stenosis (ECAS). Wehereby sought to explore the relationship of Lp-PLA $A_{2}$ and ICAS, ECAS and concurrent stenosis in stroke-free hypertensive patients in Chinese population.

\section{Methods}

All the subjects were evaluated for the presence and severity of ICAS and ECAS through computerized tomographic angiography (CTA) covered the whole brain down to the level of aortic arch. Lp-PLA $A_{2}$ mass was measured by enzyme linked immunoassay. The association of $L p-P L A_{2}$ and vascular stenosis was analyzed through multivariate logistic regression.

\section{Results}

Among 414 participants, 163 (39.4\%) had no ICAS or ECAS, 63 (15.2\%) had ECAS only, $111(26.8 \%)$ had ICAS only and $77(18.6 \%)$ had concurrent extraintracranial stenosis. Lp$\mathrm{PLA}_{2}$ mass was significantly associated with isolated ICAS (OR: $2.3 ; 95 \% \mathrm{Cl}: 1.14-4.64$ ), and concurrent stenosis (OR: $3.93 ; 95 \% \mathrm{Cl}: 1.62-9.51$ ), but was not related to isolated ECAS (OR: $1.54 ; 95 \%$ Cl: 0.68-3.48). Lp-PLA 2 mass was also associated with moderate to severe ICAS no matter how was the ECAS. Moreover, patients with higher Lp-PLA 2 mass showed more sever ICAS and had more intracranial arterial lesions. 
Competing Interests: The authors have declared that no competing interests exist.

\section{Conclusion}

This study revealed the association of Lp-PLA 2 mass with ICAS in stroke-free hypertensive patients in Chinese population. The further long-term cohort study was warranted to elucidate the concrete effect of $L p-P L A_{2}$ on the asymptomatic ICAS.

\section{Introduction}

Stroke is a global health problem and is the leading cause of death in China [1], with ischemic stroke as the predominant subtype. Intracranial arterial stenosis (ICAS) is a more common cause of ischemic stroke in Asians, whereas whites tend to have more extracranial lesions [24]. Meanwhile, hypertension is a well-established risk factor for both ischemic and hemorrhagic forms of stroke, as well as for ICAS $[2,5,6]$. However, there is limited study on the risk factors of asymptomatic ICAS, extracranial arterial stenosis (ECAS) and concurrent stenosis in hypertension patients.

Lipoprotein-associated phospholipase $\mathrm{A}_{2}$ (Lp-PLA $\mathrm{A}_{2}$ can cleave the oxidized fatty acid side chain at the sn2 position of oxidized phospholipids, which generates lysophosphatidylcholine and oxidized free fatty acid, and mediates multiple inflammatory pathway [7, 8]. Recently, elevated circulating levels of $\mathrm{Lp}-\mathrm{PLA}_{2}$ have been shown to be independent predictors of coronary heart disease and ischemic stroke in general population and clinical patients $[9,10]$. However, there is no study focusing on the relationship of Lp-PLA $A_{2}$ and asymptomatic ICAS or ECAS. Therefore, in the present study, we aim to investigate the association of Lp- $\mathrm{PLA}_{2}$ and ICAS, ECAS and concurrent stenosis in stroke-free hypertensive patients in Chinese population.

\section{Methods}

\section{Study design}

This study was undertaken within the framework of an ongoing cross-section and prospective study in China, which was a computerized tomographic angiography (CTA) based study of intra- and extracranial asymptomatic artery stenosis and stroke outcome in stroke-free hypertension patients. Subjects of this study were recruited from hypertension outpatients who were identified in Xinzhuang Community hospital between May 2012 and May 2013, and then referred to Ruijin Hospital, a general hospital in Shanghai. Hypertensive status was defined as systolic $\mathrm{BP}(\mathrm{SBP}) \geq 140 \mathrm{mmHg}$ and/ordiastolic $\mathrm{BP}(\mathrm{DBP}) \geq 90 \mathrm{mmHg}$, or individuals taking antihypertensive medication. All the participants were over 50 years old. Those who had stroke, transient ischemic attack (TIA), or atrial fibrillation identified from medical history was excluded. Those who were unfit for CTA examination because of iodine allergy were also excluded.

\section{Ethics statement}

The study protocol was approved by the ethics committee of Ruijin Hospital and written informed consent was obtained from all participants.

\section{Demographic and clinical measurements}

After the subjects had rested for at least $5 \mathrm{~min}$ in the sitting position, SBP and DBP were measured using a verified electronic sphygmomanometer (OMRON, HEM-907) by a trained 
physician or nurse. The average of three consecutive BP readings with one minute interval of each participant was used for the current analysis. Body weight and height were recorded with participants wearing light indoor clothing and no shoes. Clinical information was collected by interview, including smoking and drinking habits, current drug intake, personal and family history of hypertension and diabetes, etc. Current smokers were defined as those who had smoked cigarettes on one or more days in the past 30 days. All the biochemical measurements including fasting plasma glucose, serum concentrations of total cholesterol (TC), triglycerides (TGs), high-density lipoprotein cholesterol (HDL), low-density lipoprotein cholesterol (LDL), serum creatinine, serum urea nitrogen, uric acid, urine albumin creatinine ratio and neutrophil accounts were performed in the Central Laboratory of Ruijin Hospital (Shanghai, China) using the standard protocols.

$\mathrm{Lp}-\mathrm{PLA}_{2}$ mass was measured in plasma aliquots stored in $-80^{\circ} \mathrm{C}$ freezers, using an enzyme linked immunoassay. Samples were incubated in microtitre plate wells with immobilized monoclonal antibody against Lp-PLA 2 (R\&D AF5106,MN, USA), and then it was identified by an anti-Lp-PLA ${ }_{2}$ antibody labeled with horseradish peroxidase. The range of detection was 40 to $1200 \mathrm{ng} / \mathrm{ml}$. Lp-PLA 2 mass was measured in duplicate with intra-assay CV of $2.6 \%$.

\section{CTA protocol}

CTA was performed with a 64-section helical CT scanner (GE FX/I, General Electric, Fairfield, CT) as our previous prescription [11]. CTA acquisitions were obtained after a single bolus intravenous injection of $70 \mathrm{ml}$ Optirayloversol 320 into the antecubital vein at a rate of $3 \mathrm{ml} / \mathrm{sec}$. Scanning covered the whole brain down to the level of aortic arch with 5-mm slice thickness. Images were reformatted in axial, sagittal, and coronal planes with $1.25-\mathrm{mm}$ slice thickness. All images were read at a workstation with the software of AW4.4 vessel analysis independently by two experienced radiologist who were blinded to clinical data of the patients. Stenosis was defined as a lesion that decreased arterial internal diameter. The percentage of stenosis was calculated as the ratio of the diameter of the diseased artery at its most severe site divided by the diameter of a nearby normal segment. The degree of stenosis was categorized into mild $(<30 \%)$, moderate $(30-69 \%)$, or severe $(>=70 \%)$. The intracranial arteries included intracranial segment of internal carotid artery and vertebral artery, basilar artery, anterior cerebral artery, middle cerebral artery and posterior cerebral artery. The greatest stenosis at an intracranial or extracranial artery was chosen as being representative for each subject. The extracranial arteries included extracranial segment of internal carotid artery and vertebral artery, external carotid artery, common carotid artery and subclavian artery. The number of arteries with stenosis for each patient was also counted. The two radiologists had good agreement in the designation of stenosis $(\kappa=0.93, \mathrm{P}<0.001)$. All disagreements were reviewed and adjudicated by a senior radiologist to reach a consensus.

\section{Statistical analysis}

For database management and statistical analysis, we used SPSS software (version 13.0; SPSS Inc., Chicago, Illinois, USA). Descriptive statistics for patients with or without ICAS and ECAS were compared using a Pearson Chi-square test for categorical variables and Student $t$ test for continuous variables. Correlation was evaluated by Spearman coefficients. Multivariate logistic regression was performed to test the association of Lp-PLA ${ }_{2}$ mass with risk of ICAS and ECAS separately in two models. The basal model was adjusted for sex and age. Further analysis was adjusted for BMI, hypertension duration, current smoking and drinking status, diabetes, LDL, HDL, plasma glucose, mean arterial pressure, heart rate, neutrophil account, urine albumin creatinine ratio, serum creatinine, anti-hypertensive treatment and statin use in addition. 
Table 1. Clinical characteristics of hypertensive patients according to location and severity of arterial stenosis.

\begin{tabular}{|c|c|c|c|c|c|c|c|}
\hline \multirow[t]{2}{*}{ Baseline characteristics } & \multirow{2}{*}{$\begin{array}{l}\text { Control } \\
\text { ICAS/ECAS } \\
\text { absent }\end{array}$} & \multicolumn{3}{|c|}{ Mild to severe stenosis } & \multicolumn{3}{|c|}{ Moderate to severe stenosis } \\
\hline & & $\begin{array}{l}\text { ECAS } \\
\text { present }\end{array}$ & ICAS present & COMB present & ECAS present & ICAS present & COMB present \\
\hline $\mathrm{N}$ & 163 & 63 & 111 & 77 & 38 & 35 & 14 \\
\hline Age (years) & $64.4 \pm 5.9$ & $65.5 \pm 5.5$ & $67.1 \pm 5.6 \dagger$ & $68.3 \pm 5.4 \dagger$ & $67.1 \pm 5.8^{*}$ & $66.7 \pm 5.7^{*}$ & $68.9 \pm 5.6 \dagger$ \\
\hline Male $(\mathrm{N}, \%)$ & $60(36.8)$ & $32(50.8)$ & $60(54.1) \dagger$ & $44(57.1) \dagger$ & $13(34.2)$ & $15(42.90)$ & $5(57.1)$ \\
\hline Smoking (N,\%) & $17(10.4)$ & $12(19)$ & $20(18)$ & $16(20.8)$ & $9(23.7)^{*}$ & $9(25.7)^{*}$ & $2(14.3)$ \\
\hline Dringking $(\mathrm{N}, \%)$ & $24(14.7)$ & $9(14.3)$ & $18(16.2)$ & $10(13)$ & $6(15.8)$ & $5(14.3)$ & $2(14.3)$ \\
\hline Diabetes mellitus ( $\mathrm{N}, \%)$ & $38(23.3)$ & $9(14.3)$ & $33(29.7)$ & $26(33.8)$ & $7(18.4)$ & $17(48.6) \dagger$ & $6(42.9) \dagger$ \\
\hline $\begin{array}{l}\text { Hypertension duration } \\
\text { (years) }\end{array}$ & $11(5-20)$ & $10(3-16)$ & $12(8-22)^{*}$ & $15(8-22)^{*}$ & $8.0(3.8-15.3)$ & $\begin{array}{l}20.1(10.4- \\
29.2) \dagger\end{array}$ & $15.0(5.8-17.3)$ \\
\hline $\begin{array}{l}\text { Anti-hypertensive treatment } \\
(\mathrm{N}, \%)\end{array}$ & $146(89.6)$ & $56(88.9)$ & 103(92.8) & $65(84.4)$ & $31(81.6)$ & $32(91.4)$ & $11(78.6)$ \\
\hline Statin use $(\mathrm{N}, \%)$ & $6(3.7)$ & $6(9.5)$ & $5(4.5)$ & $4(5.2)$ & $3(7.9)$ & $3(8.6)$ & $1(7.0)$ \\
\hline Body mass index (kg/m2) & $24.8 \pm 3.7$ & $25.3 \pm 3$ & $25.1 \pm 2.8$ & $25.3 \pm 2.9$ & $24.8 \pm 2.5$ & $25.4 \pm 3.4$ & $25.1 \pm 2.8$ \\
\hline $\begin{array}{l}\text { Systolic blood pressure } \\
(\mathrm{mmHg})\end{array}$ & $136.3 \pm 16.5$ & $139.4 \pm 15.3$ & $142.6 \pm 16.6 \dagger$ & $143.9 \pm 19.9$ & $140.1 \pm 17.2$ & $141 \pm 18.3$ & $149.6 \pm 19.6 \dagger$ \\
\hline $\begin{array}{l}\text { Diastolic blood pressure } \\
(\mathrm{mmHg})\end{array}$ & $73 \pm 9.9$ & $72.4 \pm 10.2$ & $74.2 \pm 10.9$ & $71.4 \pm 10.3$ & $71.3 \pm 9.4$ & $70.8 \pm 9.9$ & $72 \pm 11.4$ \\
\hline Heart rate (beats/min) & $76.4 \pm 13.2$ & $74.4 \pm 12.1$ & $73.4 \pm 10.2^{*}$ & $74.6 \pm 10.4$ & $74.8 \pm 11$ & $70.5 \pm 10^{*}$ & $79 \pm 12.9$ \\
\hline Plasma glucose (mmol/L) & $5.1 \pm 1.4$ & $4.8 \pm 0.7$ & $5.1 \pm 1$ & $5.5 \pm 1.4^{*}$ & $5 \pm 1.2$ & $5.5 \pm 1.3$ & $5.8 \pm 1.7$ \\
\hline Total cholesterol (mmol/L) & $4.8 \pm 0.8$ & $5 \pm 0.8$ & $4.8 \pm 0.9$ & $5 \pm 0.8$ & $5 \pm 0.8$ & $4.8 \pm 0.7$ & $5.4 \pm 0.6^{*}$ \\
\hline LDL (mmol/L) & $2.9 \pm 0.7$ & $3.1 \pm 0.7$ & $2.9 \pm 0.8$ & $3.2 \pm 0.8 \dagger$ & $3.2 \pm 0.7^{*}$ & $3 \pm 0.8$ & $3.5 \pm 0.6 \dagger$ \\
\hline $\mathrm{HDL}(\mathrm{mmol} / \mathrm{L})$ & $1.2 \pm 0.3$ & $1.2 \pm 0.2$ & $1.2 \pm 0.3$ & $1.1 \pm 0.2^{*}$ & $1.2 \pm 0.2$ & $1.1 \pm 0.2 \dagger$ & $1.1 \pm 0.1$ \\
\hline Triglyceride (mmol/L) & $1.6 \pm 0.8$ & $1.6 \pm 0.8$ & $1.7 \pm 0.9$ & $1.7 \pm 0.8$ & $1.6 \pm 0.8$ & $1.9 \pm 0.8$ & $1.4 \pm 0.7$ \\
\hline Serum creatinine (mmol/L) & $65.3 \pm 19.8$ & $68.3 \pm 18.9$ & $70.2 \pm 19.1^{*}$ & $69.5 \pm 20.3$ & $69.2 \pm 16.7$ & $72.7 \pm 20.0^{*}$ & $60.6 \pm 14.4$ \\
\hline $\begin{array}{l}\text { Serum urea nitrogen }(\mathrm{mmol} / \\
\text { L) }\end{array}$ & $5.6 \pm 1.3$ & $5.7 \pm 1.4$ & $5.7 \pm 1.3$ & $5.5 \pm 1.3$ & $5.4 \pm 1.3$ & $5.6 \pm 1.2$ & $5.6 \pm 1.4$ \\
\hline Uric acid (mmol/L) & $321.3 \pm 71.8$ & $332.5 \pm 70.8$ & $335.2 \pm 73.9$ & $333.4 \pm 75.5$ & $325.8 \pm 73.5$ & $346.5 \pm 77.3$ & $309.6 \pm 67.6$ \\
\hline ACR $(\mathrm{mg} / \mathrm{mmol})$ & $3.1(1.8-6.0)$ & $2.8(1.9-3.9)$ & $3.8(2.0-6.7)$ & $3.9(2.3-7.8)$ & $3.0(1.8-5.3)$ & $4.3(2.5-6.3)$ & $3.9(2.9-10.3)$ \\
\hline Neutrophil account $\left({ }^{*} 109 / L\right)$ & $3.6(2.9-4.4)$ & $3.7(3.0-4.5)$ & $3.6(3.0-4.6)$ & $3.9(3.3-4.7)^{*}$ & $4.1(3.5-4.0)$ & $4.3(3.2-5.2)^{*}$ & $3.4(3.2-4.0)$ \\
\hline Lp-PLA 2 (ng/ml) & $\begin{array}{l}206.6(109.8- \\
340.8)\end{array}$ & $\begin{array}{l}217(118.4- \\
408.8)\end{array}$ & $\begin{array}{l}249.6(131.8- \\
430.2)^{*}\end{array}$ & $\begin{array}{l}266.2(152.4- \\
443.1) \dagger\end{array}$ & $\begin{array}{l}239.2(10.8 .0- \\
415.0)\end{array}$ & $\begin{array}{l}266.2(154.8- \\
461.6)^{*}\end{array}$ & $\begin{array}{l}309.3(266.4- \\
536.9) \dagger\end{array}$ \\
\hline
\end{tabular}

Data are expressed as mean \pm SD, median (interquartile range), or percentage (\%). Each group was compared with the control group using $\chi 2$ or Student $t$ test. ECAS, extracranial arterial stenosis; ICAS, intracranial arterial stenosis; COMB, combined extra- and intracranial arterial stenosis. LDL, low-density lipoprotein; HDL, high-density lipoprotein;ACR, urine albumin creatinine ratio Lp-PLA, Lipoprotein-associated phospholipase A2. In the comparison among groups, hypertension duration, $A C R$ and $L p-P L A_{2}$ are log-transformed. ICAS/ECAS absent group is considered as control.

$* p<0.05$

$\dagger p<0.01$

doi:10.1371/journal.pone.0130473.t001

Logarithmic transformation was used on variables that were not normally distributed. All $P$ values were 2 -tailed, and a $P$ value of $<0.05$ was considered statistically significant.

\section{Results}

The general characteristics of patients according to the location and severity of stenosis were shown in Table 1. Of 414 subjects included in the study, 39.4\% had no ICAS or ECAS, 15.2\% had ECAS only, $26.8 \%$ had ICAS only, and $18.6 \%$ had concurrent extraintracranial artery stenosis. Among the 67 subjects with moderate to severe stenosis, $60.3 \%$ had ECAS only, 31.5\% 
Table 2. Associations of Lp-PLA 2 mass with intracranial and extracranial arterial stenosis.

\begin{tabular}{|c|c|c|c|c|c|c|}
\hline & \multicolumn{3}{|c|}{ Model I } & \multicolumn{3}{|c|}{ Model II } \\
\hline & OR & $95 \% \mathrm{Cl}$ & $\mathbf{P}$ & OR & $95 \% \mathrm{Cl}$ & $\mathbf{P}$ \\
\hline Isolated ICAS & 2.30 & $1.14-4.64$ & 0.020 & 2.27 & $1.11-4.65$ & 0.025 \\
\hline Isolated ECAS & 1.54 & $0.68-3.48$ & 0.301 & 1.3 & $0.5-3.35$ & 0.589 \\
\hline COMB & 3.93 & $1.62-9.51$ & 0.002 & 3.73 & $1.24-11.25$ & 0.020 \\
\hline Complex ICAS & 2.92 & $1.54-5.54$ & 0.001 & 2.97 & $1.36-6.48$ & 0.006 \\
\hline Isolated moderate to severe ICAS & 3.63 & $1.16-11.30$ & 0.026 & 3.17 & $0.68-14.70$ & 0.140 \\
\hline Isolated moderate to severe ECAS & 1.17 & $0.44-3.14$ & 0.757 & 0.96 & $0.30-3.12$ & 0.950 \\
\hline moderate to severe $\mathrm{COMB}$ & 15.39 & $2.03-116.55$ & 0.008 & 13.41 & $1.72-104.42$ & 0.013 \\
\hline Complex moderate to severe ICAS & 3.57 & $1.37-9.33$ & 0.009 & 3.53 & $1.32-9.46$ & 0.012 \\
\hline
\end{tabular}

OR, odds ratio. Model I adjusted for age and sex. Model II adjusted for age, sex, BMI, hypertension duration, current smoking and drinking status, diabetes, LDL, HDL, plasma glucose, mean arterial pressure, heart rate, neutrophil account, urine albumin creatinine ratio, serum creatinine, antihypertensive treatment, and statin use. The odds ratio expressed the risk in the ICAS and ECAS group compared with the non-stenosis group. Isolated ECAS, extracranial arterial stenosis only; Isolated ICAS, intracranial arterial stenosis only; COMB, combined extra- and intracranial arterial stenosis. Complex ICAS, intracranial arterial stenosis no matter how was the extracranial arteries; Hypertension duration, neutrophil account, serum creatinine, urine albumin creatinine ratio and $\mathrm{Lp}-\mathrm{PLA} \mathrm{A}_{2}$ are log-transformed.

doi:10.1371/journal.pone.0130473.t002

had ICAS only, and $18.2 \%$ had concurrent stenosis. Comparing with the stenosis absent group, the patients with ICAS were older and with higher frequency of male, longer hypertension duration, higher SBP, slower heart rates, higher serum creatinine and Lp-PLA 2 mass. And the patients with moderate to severe ICAS were more likely to be smoking, with the comorbidity of diabetes and lower HDL.

\section{Lp-PLA $A_{2}$ ass and ICAS/ECAS}

In models only adjusted with sex and age, the Lp-PLA 2 mass was significantly associated with isolated ICAS (OR: 2.3; 95\% CI: 1.14-4.64), and concurrent extraintracranial stenosis (OR: 3.93; 95\% CI: 1.62-9.51) (Table 2). Considering that Lp-PLA 2 mass was not related to isolated ECAS (OR: 1.54; 95\% CI: 0.68-3.48), we further estimated the relationship of Lp-PLA $\mathrm{P}_{2}$ mass and complex ICAS, which referred to ICAS no matter how the extracranial arteries was. A significant association was found between Lp-PLA 2 mass and complex ICAS (OR: 2.92; 95\% CI: 1.54-5.54). After additional adjustment for other risk factors, including BMI, hypertension duration, current smoking and drinking status, diabetes, LDL, HDL, plasma glucose, mean arterial pressure, heart rate, neutrophil account, urine albumin creatinine ratio, serum creatinine, anti-hypertensive treatment and statin use, the strength of association attenuated but was still significant.

\section{Lp-PLA 2 mass and severity/location of ICAS/ECAS}

Similarly, in simple adjusted logistic regression analysis, Lp-PLA 2 mass were significantly associated with isolated moderate to severe ICAS (OR: 3.63; 95\% CI: 1.16-11.3), concurrent moderate to severe extraintracranial stenosis (OR: 15.39; 95\% CI: 2.03-116.55), and complex moderate to severe ICAS (OR: 3.57; 95\% CI: 1.37-9.33). However, after multivariate adjusted logistic analysis, only concurrent stenosis and complex ICAS showed association with Lp-PLA 2 mass, which might result from the relative small amount of moderate to severe ICAS.

Compared to the subjects in the lowest tertile of Lp-PLA 2 mass (11.5\%), more subjects in tertile $2(13.7 \%)$ and tertile $3(16.1 \%)$ suffered moderate to severe ICAS $(\mathrm{P}<0.05)$ (Fig $1 \mathrm{~A})$. 


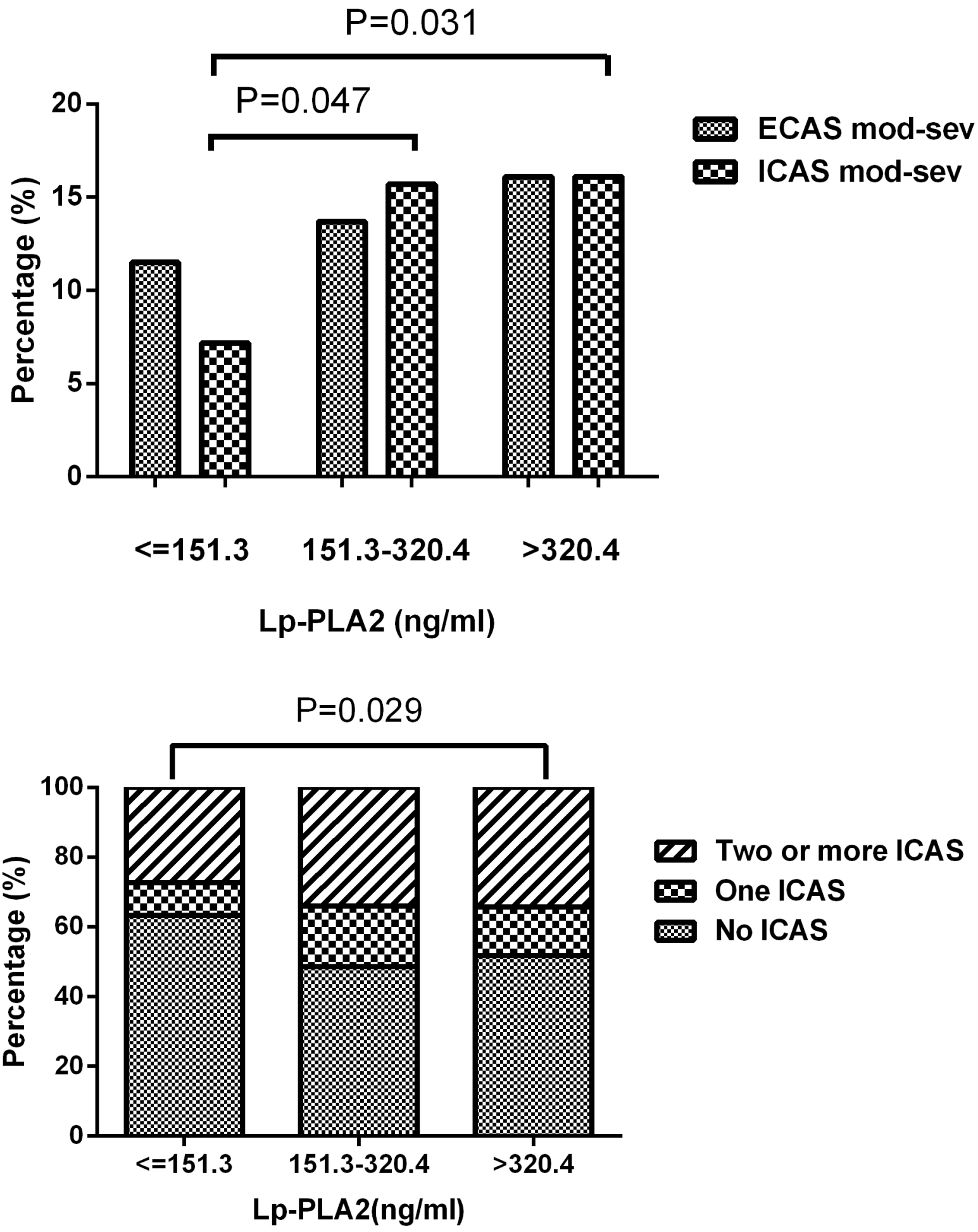

Fig 1. Prevalence of moderate to severe ECAS and ICAS (\%) (A) and distribution of ICAS vessels (B) according to tertiles of Lp-PLA . ECAS, extracranial arterial stenosis; ICAS, intracranial arterial stenosis; Lp-PLA2, Lipoprotein-associated phospholipase $A_{2}$.

doi:10.1371/journal.pone.0130473.g001 
Table 3. Associations of Lp-PLA 2 mass with complex intracranial arterial stenosis in subgroup analysis.

\begin{tabular}{llll}
\hline & OR & $\mathbf{9 5 \%}$ Cl & P \\
\hline Men & 0.99 & $0.64-6.18$ & 0.233 \\
Women & 5.00 & $1.47-17.09$ & $\mathbf{0 . 0 1 0}$ \\
Age $<=65.8 \mathrm{y}$ & 3.46 & $1.33-8.98$ & $\mathbf{0 . 0 1 1}$ \\
Age $>65.8 \mathrm{y}$ & 2.42 & $1.01-5.81$ & $\mathbf{0 . 0 4 7}$ \\
$\mathrm{LDL}<=3.0 \mathrm{mmol} / \mathrm{L}$ & 2.47 & $0.84-7.26$ & 0.100 \\
$\mathrm{LDL}>3.0 \mathrm{mmol} / \mathrm{L}$ & 5.29 & $1.43-19.5$ & $\mathbf{0 . 0 1 2}$ \\
\hline
\end{tabular}

doi:10.1371/journal.pone.0130473.t003

Moreover, patients in the highest tertile of Lp-PLA 2 mass showed higher frequency of multiple ICAS (34.3\%) than the lowest tertile group (27.3\%) (Fig 1B). The severity or number of ECAS was not related to the Lp-PLA 2 mass level.

\section{Sensitivity test}

We also checked the consistency of our results for complex ICAS according to various baseline characteristics (Table 3). After the subjects were subdivided according to median of age (65.8y), the magnitude of the association was similar among groups. The results for women and subjects with $\mathrm{LDL}>3.0 \mathrm{mmol} / \mathrm{L}$ were similar to the results of combined analyses of data. However, in men and subjects with $\mathrm{LDL}<=3.0 \mathrm{mmol} / \mathrm{L}$ group, the association of $\mathrm{Lp}^{-\mathrm{PLA}_{2}}$ mass and ICAS was not significant.

\section{Discussion}

In this stroke-free Chinese hypertension population who underwent both intra- and extracranial CTA, a significant and independent association was found of Lp-PLA 2 mass with ICAS and concurrent stenosis. No significant difference of Lp-PLA $A_{2}$ mass was detected among patients with or without ECAS. Moreover, subjects with higher Lp-PLA ${ }_{2}$ mass showed more sever ICAS and had more intracranial arterial lesions.

As a minimally invasive imaging modality, CTA had been proved to provide better delineation of the anatomy of intra- and extracranial arteries [12,13], thus yielding higher diagnostic accuracy of the luminal stenosis of ICAS as compared with TCD and MRA, with DSA as the reference standard [14] In the present study, we evaluated the stenosis of both intra- and extracranial arteries by CTA, which could increase the sensitivity and accuracy of stenosis estimation.

Although the vascular risk factors were similar in the process of atherogenesis, the distribution and severity of atherogenesis varied among different individuals, whereas patients of Asian, African and Hispanic ancestry were at high risk of ICAS [15], while whites more frequently suffered from excranial carotid lesions [16]. Several previous studies had showed the different risk factors for ICAS and ECAS. In a study including 425 stroke-free Japanese patients, Uehara et al. found that the independent predictors of ECAS were age, hyperlipidemia and ischemic heart disease (IHD), while those for ICAS were age, hypertension, diabetes mellitus and IHD [17]. The study of Lopez-Cancio et al. in a Spanish population found that male sex, hypertension, smoking were independent risk factors of isolated ECAS, and diabetes and metabolic syndrome conferred a higher risk for ICAS [18]. Similar with the former studies, we found that the subjects with isolated ECAS were younger, and showed higher TC, lower SBP and shorter hypertension duration than those with isolated ICAS. 
Since the first report in 2000 [19], a great deal of transectional and prospective studies had investigated the association between circulating Lp- $\mathrm{PLA}_{2}$ and ischemic stroke. In a populationbased study, Oei et al. found the subjects with the fourth quartile of Lp-PLA2 activity were at the high risk for ischemic stroke [9]. The Atherosclerosis Risk in Communities Study underwent a case-control study and found that Lp-PLA ${ }_{2}$ and hs-CRP were useful intermediate risk factor for ischemic stroke in addition to traditional factor [20]. The meta-analysis performed by Lp-PLA ${ }_{2}$ studies collaboration, including 79036 participants in 32 prospective studies confirmed the association of Lp-PLA 2 mass with ischemic stroke [21]. A study of symptomatic patient population also found Lp-PLA 2 activity was increased in patients with multiple or bilateral stenosis [22].

We hereby reported a significant and independent association of Lp-PLA 2 mass with ICAS, but not with ECAS. The reasons that explained the different effect of $\mathrm{Lp}-\mathrm{PLA}_{2}$ on the distribution of atherosclerotic lesions were not well known. Even though it was the case that atherosclerosis was a systemic disease and the vascular system is uniformly exposed to risk factors, the inflammatory responds seemed differentially regulated in certain site-specific pattern. Mohler et al. had observed the lesions development of coronary arteries, thoracic aorta and carotid arteries in swine for nine months, in which they found expression of $\mathrm{Lp}-\mathrm{PLA}_{2}$ was unregulated only in coronary but not carotid arteries [23]. Rotterdam Study also showed Lp-PLA $\mathrm{A}_{2}$ was not associated with carotid arteries atherosclerosis [24], which was in accordance with our findings. Several theories provided cues for the site specific impact of risk factors on arteries, including different anatomical origins [25], various shear stress dependent endothelial gene expressions [26], and diverse shear stress dependent accumulation of inflammatory cells in specific vascular regions [27]. Furthermore, the additional risk factors might also influence the effect of Lp$\mathrm{PLA}_{2}$ on extraintracranial stenosis. In this study, all the subjects had the history of hypertension, which probably act synergistically with Lp-PLA 2 during the formation of ICAS.

In the sensitivity analysis, we found the association of ICAS and Lp-PLA ${ }_{2}$ only existed in women and patients with higher LDL. Researchers had suggested that the relationship between Lp-PLA ${ }_{2}$ and cardiovascular diseases might be influenced by the level of plasma lipid. In a study with 580 hyperlipidemic men, Lp-PLA ${ }_{2}$ was approved to be a strong predictor of coronary heart disease [19]. Contrarily, the study performed in healthy middle-aged women with relatively low plasma lipid achieved opposite result [28]. In our population, most of the women were over 60 years old, and displayed higher LDL than men. It might be the LDL level but not the gender which could impact the effect of Lp-PLA 2 on ICAS.

Several limitations of our study should be considered. First, we measured Lp-PLA 2 mass only, which might miss some information about Lp-PLA ${ }_{2}$ activity and ICAS or ECAS. But, it was reported that the correlation between Lp-PLA ${ }_{2}$ mass and Lp-PLA $A_{2}$ activity was about 0.5 [21]. Moreover, the Lp-PLA ${ }_{2}$ mass and Lp-PLA ${ }_{2}$ activity had similar predictive power for the cardiac death [29] and stroke [30]. The American Association of Clinical Endocrinologists' (AACE) also recognized that Lp-PLA 2 mass over $223 \mathrm{ng} / \mathrm{ml}$ could be considered as a part of a global risk assessment strategy for patients with dyslipidemia and other major cardiovascular disease risk factors [31]. The Second, we didn't measure the stability of atherosclerosis plaque in extraintracranial arteries, which had been considered as an important index for prediction

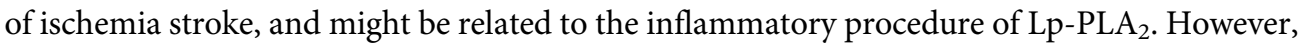
researchers had proved that ICAS rather than carotid plaque played more significant role in stroke incidence and recurrence in blacks, Hipanics and Asians [32]. Evidence from the Chinese population also indicated that carotid atherosclerosis was not independently correlated with ICAS [33]. The association of Lp-PLA ${ }_{2}$ with ICAS we found here might give some clues to understand the process from ICAS to stroke, especially in Asians. The third, patients under 
current study had a relative low cardiovascular risk profile and had few sever ICAS, which might therefore not be directly generalized to high risk patients.

In conclusion, this study revealed an association between Lp-PLA 2 mass and ICAS, but not ECAS, in stroke-free hypertension patients of Chinese population. Patients with higher Lp$\mathrm{PLA}_{2}$ mass showed more sever ICAS and had more intracranial arterial lesions. Studies based on Lp-PLA ${ }_{2}$ activity and other inflammatory measurements were suggested. In addition, the further long-term cohort study was warranted to elucidate the concrete effect of Lp-PLA ${ }_{2}$ on the asymptomatic ICAS.

\section{Author Contributions}

Conceived and designed the experiments: DZ PG. Performed the experiments: YW JZ XT YQ HL KC. Analyzed the data: YW. Contributed reagents/materials/analysis tools: HL KC. Wrote the paper: YW DZ.

\section{References}

1. He J, Gu D, Wu X, Reynolds K, Duan X, Yao C, et al. Major causes of death among men and women in china. N Engl J Med. 2005; 353:1124-1134 PMID: 16162883

2. Huang YN, Gao S, Li SW, Huang Y, Li JF, Wong KS, et al. Vascular lesions in chinese patients with transient ischemic attacks. Neurology. 1997; 48:524-525 PMID: 9040750

3. Liu HM, Tu YK, Yip PK, Su CT. Evaluation of intracranial and extracranial carotid steno-occlusive diseases in taiwanchinese patients with mr angiography: Preliminary experience. Stroke. 1996; 27:650653 PMID: 8614924

4. Wong KS, Li H, Chan YL, Ahuja A, Lam WW, Wong A, et al. Use of transcranial doppler ultrasound to predict outcome in patients with intracranial large-artery occlusive disease. Stroke. 2000; 31:26412647 PMID: 11062288

5. Bae HJ, Lee J, Park JM, Kwon O, Koo JS, Kim BK, et al. Risk factors of intracranial cerebral atherosclerosis among asymptomatics. Cerebrovasc Dis. 2007; 24:355-360 PMID: 17690548

6. Chen CT, Li Y, Zhang J, Wang Y, Ling HW, Chen KM, et al. Association between ambulatory systolic blood pressure during the day and asymptomatic intracranial arterial stenosis. Hypertension. 2014; 63:61-67 doi: 10.1161/HYPERTENSIONAHA.113.01838 PMID: 24144646

7. Ryu SK, Mallat Z, Benessiano J, Tedgui A, Olsson AG, Bao W, et al. Phospholipase a2 enzymes, highdose atorvastatin, and prediction of ischemic events after acute coronary syndromes. Circulation. 2012; 125:757-766 doi: 10.1161/CIRCULATIONAHA.111.063487 PMID: 22230483

8. Zalewski A, Macphee C. Role of lipoprotein-associated phospholipase a2 in atherosclerosis: Biology, epidemiology, and possible therapeutic target. ArteriosclerThrombVasc Biol. 2005; 25:923-931 PMID: 15731492

9. Oei HH, van der Meer IM, Hofman A, Koudstaal PJ, Stijnen T, Breteler MM, et al. Lipoprotein-associated phospholipase a2 activity is associated with risk of coronary heart disease and ischemic stroke: The rotterdam study. Circulation. 2005; 111:570-575 PMID: 15699277

10. Jenny NS, Solomon C, Cushman M, Tracy RP, Nelson JJ, Psaty BM, et al. Lipoprotein-associated phospholipase a(2) (Ip-pla(2)) and risk of cardiovascular disease in older adults: Results from the cardiovascular health study. Atherosclerosis. 2010; 209:528-532 doi: 10.1016/j.atherosclerosis.2009.09. 021 PMID: 19804884

11. Zhang J, Li Y, Wang $Y$, Niu W, Zhang $Y$, Gao $P$, et al. Arterial stiffness and asymptomatic intracranial large arterial stenosis and calcification in hypertensive chinese. Am J Hypertens. 2011; 24:304-309 doi: 10.1038/ajh.2010.246 PMID: 21164493

12. Anderson GB, Ashforth R, Steinke DE, Ferdinandy R, Findlay JM. Ct angiography for the detection and characterization of carotid artery bifurcation disease. Stroke. 2000; 31:2168-2174 PMID: 10978047

13. Nguyen-Huynh MN, Wintermark M, English J, Lam J, Vittinghoff E, Smith WS, Johnston SC. How accurate is ct angiography in evaluating intracranial atherosclerotic disease? Stroke. 2008; 39:1184-1188 doi: 10.1161/STROKEAHA.107.502906 PMID: 18292376

14. Leng $X$, Wong KS, Liebeskind $D S$. Evaluating intracranial atherosclerosis rather than intracranial stenosis. Stroke. 2014; 45:645-651 doi: 10.1161/STROKEAHA.113.002491 PMID: 24399377

15. Caplan LR, Gorelick PB, Hier DB. Race, sex and occlusive cerebrovascular disease: A review. Stroke. 1986; 17:648-655 PMID: 3526645 
16. Hsia DC, Moscoe LM, Krushat WM. Epidemiology of carotid endarterectomy among medicare beneficiaries: 1985-1996 update. Stroke. 1998; 29:346-350 PMID: 9472872

17. Uehara T, Tabuchi M, Mori E. Risk factors for occlusive lesions of intracranial arteries in stroke-free japanese. Eur J Neurol. 2005; 12:218-222 PMID: 15693812

18. Lopez-Cancio E, Galan A, Dorado L, Jimenez M, Hernandez M, Millan M, et al. Biological signatures of asymptomatic extra- and intracranial atherosclerosis: The barcelona-asia (asymptomatic intracranial atherosclerosis) study. Stroke. 2012; 43:2712-2719 PMID: 22798326

19. Packard CJ, O'Reilly DS, Caslake MJ, McMahon AD, Ford I, Cooney J, et al. Lipoprotein-associated phospholipase a2 as an independent predictor of coronary heart disease. West of scotland coronary prevention study group. N Engl J Med. 2000; 343:1148-1155 PMID: 11036120

20. Nambi V, Hoogeveen RC, Chambless L, Hu Y, Bang H, Coresh J, et al. Lipoprotein-associated phospholipase a2 and high-sensitivity c-reactive protein improve the stratification of ischemic stroke risk in the atherosclerosis risk in communities (aric) study. Stroke. 2009; 40:376-381 doi: 10.1161/ STROKEAHA.107.513259 PMID: 19095974

21. Thompson A, Gao P, Orfei L, Watson S, Di Angelantonio E, Kaptoge S, et al. Lipoprotein-associated phospholipase a(2) and risk of coronary disease, stroke, and mortality: Collaborative analysis of 32 prospective studies. Lancet. 2010; 375:1536-1544 doi: 10.1016/S0140-6736(10)60319-4 PMID: 20435228

22. Massot A, Pelegri D, Penalba A, Arenillas J, Boada C, Giralt D, et al. Lipoprotein-associated phospholipase a2 testing usefulness among patients with symptomatic intracranial atherosclerotic disease. Atherosclerosis. 2011; 218:181-187 doi: 10.1016/j.atherosclerosis.2011.04.031 PMID: 21620406

23. Mohler ER 3rd, Sarov-Blat L, Shi Y, Hamamdzic D, Zalewski A, Macphee C, et al. Site-specific atherogenic gene expression correlates with subsequent variable lesion development in coronary and peripheral vasculature. ArteriosclerThrombVasc Biol. 2008; 28:850-855 doi: 10.1161/ATVBAHA.107.154534 PMID: 18276914

24. Kardys I, Oei HH, van der Meer IM, Hofman A, Breteler MM, et al. Lipoprotein-associated phospholipase a2 and measures of extracoronary atherosclerosis: The rotterdam study. ArteriosclerThrombVasc Biol. 2006; 26:631-636

25. Luo X, Yang Y, Cao T, Li Z. Differences in left and right carotid intima-media thickness and the associated risk factors. ClinRadiol. 2011; 66:393-398 doi: 10.1016/j.crad.2010.12.002 PMID: 21324442

26. Resnick N, Yahav H, Khachigian LM, Collins T, Anderson KR, Dewey FC, et al. Endothelial gene regulation by laminar shear stress. AdvExp Med Biol. 1997; 430:155-164 PMID: 9330726

27. Honda HM, Hsiai T, Wortham CM, Chen M, Lin H, Navab M, et al. A complex flow pattern of low shear stress and flow reversal promotes monocyte binding to endothelial cells. Atherosclerosis. 2001; 158:385-390 PMID: 11583717

28. Blake GJ, Dada N, Fox JC, Manson JE, Ridker PM. A prospective evaluation of lipoprotein-associated phospholipase a(2) levels and the risk of future cardiovascular events in women. J Am CollCardiol. 2001; 38:1302-1306 PMID: 11691499

29. Rallidis LS, Tellis CC, Lekakis J, Rizos I, Varounis C, Charalampopoulos A, et al. Lipoprotein-associated phospholipase a(2) bound on high-density lipoprotein is associated with lower risk for cardiac death in stable coronary artery disease patients: A 3-year follow-up. J Am CollCardiol. 2012; 60:20532060 doi: 10.1016/j.jacc.2012.06.057 PMID: 23083783

30. Cucchiara BL, Messe SR, Sansing L, MacKenzie L, Taylor RA, Pacelli J, et al. Lipoprotein-associated phospholipase a2 and c-reactive protein for risk-stratification of patients with tia. Stroke. 2009; 40:2332-2336 doi: 10.1161/STROKEAHA.109.553545 PMID: 19461040

31. Jellinger PS, Smith DA, Mehta AE, Ganda O, Handelsman Y, Rodbard HW, et al. American association of clinical endocrinologists' guidelines for management of dyslipidemia and prevention of atherosclerosis. EndocrPract. 2012; 18Suppl 1:1-78 PMID: 22522068

32. White H, Boden-Albala B, Wang C, Elkind MS, Rundek T, Wright CB, et al. Ischemic stroke subtype incidence among whites, blacks, and hispanics: The northern manhattan study. Circulation. 2005: 111:1327-1331 PMID: 15769776

33. Leng XY, Chen XY, Chook P, Xiong L, Lin WH, Liu JY, et al. Correlation of large artery intracranial occlusive disease with carotid intima-media thickness and presence of carotid plaque. Stroke. 2013; 44:68-72 doi: 10.1161/STROKEAHA.112.675652 PMID: 23168454 